\title{
Infinitely many solutions for impulsive nonlinear fractional boundary value problems
}

\author{
Shapour Heidarkhani ${ }^{1 *}$, Amjad Salari ${ }^{1}$ and Giuseppe Caristi ${ }^{2}$
}

"Correspondence:

s.heidarkhani@razi.ac.ir

'Department of Mathematics,

Faculty of Sciences, Razi University,

Kermanshah, 67149, Iran

Full list of author information is

available at the end of the article

\begin{abstract}
Based on variational methods and critical point theory, the existence of infinitely many classical solutions for impulsive nonlinear fractional boundary value problems is ensured.

MSC: Primary 34A08; secondary 34B37; 58E05; 58E30; 26A33

Keywords: classical solution; infinitely many solutions; fractional differential equation; impulsive condition; variational methods; critical point theory
\end{abstract}

\section{Introduction}

This paper aims at ensuring the existence of infinitely many classical solutions for the following impulsive nonlinear fractional boundary value problem:

$$
\begin{aligned}
&{ }_{t} D_{T}^{\alpha}\left({ }_{0}^{c} D_{t}^{\alpha} u(t)\right)+a(t) u(t)=\lambda f(t, u(t))+h(u(t)), \quad t \neq t_{j}, \text { a.e. } t \in[0, T], \\
&\left(D_{\lambda, \mu}\right) \quad \Delta\left({ }_{t} D_{T}^{\alpha-1}\left({ }_{0}^{c} D_{t}^{\alpha} u\right)\right)\left(t_{j}\right)=\mu I_{j}\left(u\left(t_{j}\right)\right), \quad j=1, \ldots n, \\
& \\
& u(0)=u(T)=0,
\end{aligned}
$$

where $\alpha \in(1 / 2,1], a \in \mathrm{C}([0, T])$ such that there are two positive constants $a_{1}$ and $a_{2}$ such that $0<a_{1} \leq a(t) \leq a_{2}, \lambda>0, \mu \geq 0, f:[0, T] \times \mathbb{R} \rightarrow \mathbb{R}$ is an $\mathrm{L}^{1}$-Carathéodory function, $h: \mathbb{R} \rightarrow \mathbb{R}$ is a Lipschitz continuous function with the Lipschitz constant $L>0$, i.e.,

$$
\left|h\left(x_{1}\right)-h\left(x_{2}\right)\right| \leq L\left|x_{1}-x_{2}\right|
$$

for every $x_{1}, x_{2} \in \mathbb{R}$ and $h(0)=0,0=t_{0}<t_{1}<\cdots<t_{n}<t_{n+1}=T, \Delta\left({ }_{t} D_{T}^{\alpha-1}\left({ }_{0}^{c} D_{t}^{\alpha} u\right)\right)\left(t_{j}\right)=$ ${ }_{t} D_{T}^{\alpha-1}\left({ }_{0}^{c} D_{t}^{\alpha} u\right)\left(t_{j}^{+}\right)-{ }_{t} D_{T}^{\alpha-1}\left({ }_{0}^{c} D_{t}^{\alpha} u\right)\left(t_{j}^{-}\right)$, and ${ }_{t} D_{T}^{\alpha-1}\left({ }_{0}^{c} D_{t}^{\alpha} u\right)\left(t_{j}^{+}\right)=\lim _{t \rightarrow t_{j}^{+}}\left({ }_{t} D_{T}^{\alpha-1}\left({ }_{0}^{c} D_{t}^{\alpha} u\right)(t)\right)$ and ${ }_{t} D_{T}^{\alpha-1}\left({ }_{0}^{c} D_{t}^{\alpha} u\right)\left(t_{j}^{-}\right)=\lim _{t \rightarrow t_{j}^{-}}\left({ }_{t} D_{T}^{\alpha-1}\left({ }_{0}^{c} D_{t}^{\alpha} u\right)(t)\right)$ and $I_{j}: \mathbb{R} \rightarrow \mathbb{R}, j=1, \ldots, n$, are continuous functions.

Fractional differential equations (FDEs) are a simplification of ordinary differential equations and integration to arbitrary non-integer orders. FDEs have recently established themselves as precious tools in modeling many events in different fields of science and engineering. We can also observe plentiful applications in such fields as electrochemistry,

(c) 2016 Heidarkhani et al. This article is distributed under the terms of the Creative Commons Attribution 4.0 International License (http://creativecommons.org/licenses/by/4.0/), which permits unrestricted use, distribution, and reproduction in any medium, provided you give appropriate credit to the original author(s) and the source, provide a link to the Creative Commons license, and indicate if changes were made. 
chemistry, electromagnetic, mechanics, biology, electricity, economics, polymer rheology, control theory, regular variation in thermodynamics, signal and image processing, wave propagation, aerodynamics, electrodynamics of complex medium, blood flow phenomena, biophysics, viscoelasticity and damping, etc. (see [1-8]). There have also been important advances in the theory of fractional calculus and fractional ordinary and partial differential equations recently; for instance see [9-12]. Many researchers have explored the existence of solutions for nonlinear FDEs with various tools such as fixed-point theorems, the method of upper and lower solutions, critical point theory, the topological degree theory, and variational methods, for instance see [13-25]. We also cite [26], in which, Zhou and Peng were concerned with the Navier-Stokes equations with time-fractional derivative of order $\alpha \in(0,1)$. This type of equations can be used to simulate anomalous diffusion in fractal media. They established the existence and uniqueness of local and global mild solutions and proved the existence and regularity of classical solutions.

On the other hand, impulsive differential equations have become important in recent years as mathematical models of phenomena in both the physical and the social sciences. For example, many biological phenomena involving thresholds, bursting rhythm models in medicine and biology, optimal control models in economics, and frequency modulated systems, do exhibit impulsive effects. For the background and applications of the theory of impulsive differential equations to different areas, we refer the reader to the classical monograph [27]. For the general aspects of impulsive differential equations, we refer the reader to [28-32]. The existence of multiple solutions of impulsive problems has been studied also using the variational methods and critical point theory (see [33]). Both FDEs and impulsive differential equations have drawn intense attention from researchers in the last decades due to the numerous applications. The idea that combining these two classes of differential equations may yield an interesting and promising object of investigation, viz., impulsive FDEs, prompted numerous papers. For the recent developments in theory and applications of impulsive FDEs, we refer the reader to [34-36] and the references therein. Impulsive problems for fractional equations have been treated by topological methods in [37-40]. In [33, 41], based on variational methods and critical point theory the authors studied the existence and multiplicity of solutions for the problem $\left(D_{\lambda, \mu}\right)$, in the case $h(x)=0$ for all $x \in \mathbb{R}$.

We also cite [42-46] in which fractional systems have been studied. In $[45,46]$ through variational methods and critical point theory the existence of multiple solutions for coupled systems of nonlinear fractional differential equations was analyzed. In [44], using Ricceri's variational principle, the existence of one weak solution for a class of fractional differential systems was argued. In [43] employing Ricceri's variational principle, the existence of an infinite number of weak solutions for a class of impulsive fractional differential systems was guaranteed. In [42] using variational methods and critical point theory, the multiplicity results of solutions for a class of impulsive fractional differential systems was established.

Motivated by the researches above, this paper employs a smooth version of [47], Theorem 2.1, which is a more precise version of Ricceri's variational principle [48], Theorem 2.5. This is undertaken under several hypotheses on the behavior of nonlinear terms at infinity, under conditions on $f$ and impulsive terms $I_{j}, j=1, \ldots, n$, where we demonstrate the existence of definite intervals about $\lambda$ and $\mu$ in which the problem $\left(D_{\lambda, \mu}\right)$ admits a sequence of classical solutions which is unbounded in the space $\mathrm{E}^{\alpha}$ that will be introduced in the 
next section. Moreover, some of the consequences of Theorem 3.1 are outlined. Replacing the conditions at infinity of the nonlinear terms, by a similar one at zero, the same findings hold, and, besides, the sequence of classical solutions strongly converges to zero; see Theorem 3.4. Two examples of applications are pointed out (see Examples 3.1 and 3.2).

For an argument regarding the existence of infinitely many solutions for boundary value problems through Ricceri's variational principle [48], the reader may refer to [49-51]. We also refer the reader to [52-54] in which the existence of solutions to boundary value problems for FDEs has been studied.

\section{Preliminaries}

In this section, we will introduce several basic definitions, notations, lemmas, and propositions used throughout this paper.

Definition 2.1 ([5]) For a function $f$ defined on $[a, b]$ and $\alpha>0$, the left and right Riemann-Liouville fractional integrals of order $\alpha$ for the function $f$ are defined by

$$
\begin{aligned}
& { }_{a} D_{t}^{-\alpha} f(t)=\frac{1}{\Gamma(\alpha)} \int_{a}^{t}(t-s)^{\alpha-1} f(s) \mathrm{d} s, \quad t \in[a, b], \\
& { }_{t} D_{b}^{-\alpha} f(t)=\frac{1}{\Gamma(\alpha)} \int_{t}^{b}(s-t)^{\alpha-1} f(s) \mathrm{d} s, \quad t \in[a, b],
\end{aligned}
$$

while the right-hand sides are point-wise defined on $[a, b]$, where $\Gamma(\alpha)$ is the gamma function.

Definition 2.2 ([5]) Let $a, b \in \mathbb{R}$ and $\mathrm{AC}([a, b])$ be the space of absolutely continuous functions on $[a, b]$. For $0<\alpha \leq 1, f \in \mathrm{AC}([a, b])$ left and right Riemann-Liouville and Caputo fractional derivatives are defined by

$$
\begin{aligned}
& { }_{a} D_{t}^{\alpha} f(t) \equiv \frac{\mathrm{d}}{\mathrm{d} t}{ }_{a} D_{t}^{\alpha-1} f(t)=\frac{1}{\Gamma(1-\alpha)} \frac{\mathrm{d}}{\mathrm{d} t} \int_{a}^{t}(t-s)^{-\alpha} f(s) \mathrm{d} s, \\
& { }_{t} D_{b}^{\alpha} f(t) \equiv-\frac{\mathrm{d}}{\mathrm{d} t}{ }_{t} D_{b}^{\alpha-1} f(t)=-\frac{1}{\Gamma(1-\alpha)} \int_{t}^{b}(s-t)^{-\alpha} f(s) \mathrm{d} s, \\
& { }_{a}^{c} D_{t}^{\alpha} f(t) \equiv{ }^{c} D_{a^{+}}^{\alpha} f(t):={ }_{a} D_{t}^{\alpha-1} f^{\prime}(t)=\frac{1}{\Gamma(1-\alpha)} \int_{a}^{t}(t-s)^{-\alpha} f^{\prime}(s) \mathrm{d} s,
\end{aligned}
$$

and

$$
{ }_{t}^{c} D_{b}^{\alpha} f(t) \equiv{ }^{c} D_{b-}^{\alpha} f(t):=-{ }_{t} D_{b}^{\alpha-1} f^{\prime}(t)=-\frac{1}{\Gamma(1-\alpha)} \int_{t}^{b}(s-t)^{-\alpha} f^{\prime}(s) \mathrm{d} s,
$$

where $\Gamma(\alpha)$ is the gamma function. Note that when $\alpha=1,{ }_{a}^{c} D_{t}^{1} f(t)=f^{\prime}(t)$ and ${ }_{t}^{c} D_{b}^{1} f(t)=$ $-f^{\prime}(t)$

We have the following property of fractional integration.

Proposition 2.1 ([5, 8]) We have the following property of fractional integration:

$$
\int_{a}^{b}\left[{ }_{a} D_{t}^{-\gamma} f(t)\right] g(t) \mathrm{d} t=\int_{a}^{b}\left[{ }_{t} D_{b}^{-\gamma} g(t)\right] f(t) \mathrm{d} t, \quad \gamma>0,
$$


provided that $f \in \mathrm{L}^{p}\left([a, b], \mathbb{R}^{N}\right), g \in \mathrm{L}^{q}\left([a, b], \mathbb{R}^{N}\right)$ and $p \geq 1, q \geq 1,1 / p+1 / q \leq 1+\gamma$ or $p \neq 1, q \neq 1,1 / p+1 / q=1+\gamma$.

To create suitable function spaces and apply critical point theory to explore the existence of solutions for the problem $\left(D_{\lambda, \mu}\right)$, we require the following essential notations and findings which will be used in establishing our main results.

Let $0<\alpha \leq 1,1<p<\infty$, and $\mathrm{E}_{0}^{\alpha, p}(0, T)$ be the Banach space, which has closure of $\mathrm{C}_{0}^{\infty}([0, T])$ with respect to the norm $\|u\|_{\mathrm{E}_{0}^{\alpha, p}(0, T)}^{p}=\left\|_{a}^{c} D_{t}^{\alpha} u(t)\right\|_{\mathrm{L}^{p}(0, T)}^{p}+\|u\|_{\mathrm{L}^{p}(0, T)}^{p}$. It is an established fact that $\mathrm{E}_{0}^{\alpha, p}(0, T)$ is a reflexive and separable Banach space (see [23], Proposition 3.1). In short $\mathrm{E}_{0, T}^{\alpha, 2}=\mathrm{E}^{\alpha}$, and by $\|\cdot\|$ and $\|\cdot\|_{\infty}$ the norms in $\mathrm{L}^{2}(0, T)$ and $\mathrm{C}([0, T])$ :

$$
\begin{aligned}
& \|u\|^{2}=\int_{0}^{T}|u(t)|^{2} \mathrm{~d} t, \quad u \in \mathrm{L}^{2}(0, T), \\
& \|u\|_{\infty}=\max _{t \in[0, T]}|u(t)|, \quad u \in \mathrm{C}([0, T]) .
\end{aligned}
$$

$\mathrm{E}^{\alpha}$ is a Hilbert space with inner product $\left.(u, v)_{\alpha}=\int_{0}^{T}{ }_{0}^{c} D_{t}^{\alpha} u(t)_{0}^{c} D_{t}^{\alpha} v(t)+u(t) v(t)\right) \mathrm{d} t$ and the norm $\|u\|_{\alpha}^{2}=\int_{0}^{T}\left(\left|{ }_{0}^{c} D_{t}^{\alpha} u(t)\right|^{2}+|u(t)|^{2}\right) \mathrm{d} t$. Note that if $a \in \mathrm{C}([0, T])$ and there are two positive constants $a_{1}$ and $a_{2}$, so that $0<a_{1} \leq a(t) \leq a_{2}$, an equivalent norm in $E^{\alpha}$ is

$$
\|u\|_{a, \alpha}^{2}=\int_{0}^{T}\left(\left|{ }_{0}^{c} D_{t}^{\alpha} u(t)\right|^{2} \mathrm{~d} t+a(t)|u(t)|^{2}\right) \mathrm{d} t .
$$

Proposition 2.2 ([23]) Let $0<\alpha \leq 1$. For $u \in \mathrm{E}^{\alpha}$, we have

$$
\|u\| \leq \frac{T^{\alpha}}{\Gamma(\alpha+1)}\left\|_{0}^{c} D_{t}^{\alpha} u\right\|
$$

In addition, for $\frac{1}{2}<\alpha \leq 1$,

$$
\|u\|_{\infty} \leq \frac{T^{\alpha-1 / 2}}{\Gamma(\alpha)(2 \alpha-1)^{1 / 2}}\left\|{ }_{0}^{c} D_{t}^{\alpha} u\right\| .
$$

By (1), we can take $\mathrm{E}^{\alpha}$ with the norm

$$
\|u\|_{0, \alpha}=\left(\int_{0}^{T}\left|{ }_{0}^{c} D_{t}^{\alpha} u(t)\right|^{2} \mathrm{~d} t\right)^{1 / 2}=\left\|{ }_{0}^{c} D_{t}^{\alpha} u\right\|, \quad \forall u \in \mathrm{E}^{\alpha},
$$

in the following.

By Proposition 2.2, when $\alpha>1 / 2$, for every $u \in \mathrm{E}^{\alpha}$ we have

$$
\|u\|_{\infty} \leq k\left(\int_{0}^{T}\left|{ }_{0}^{c} D_{t}^{\alpha} u(t)\right|^{2} \mathrm{~d} t\right)^{1 / 2}=k\|u\|_{0, \alpha}<k\|u\|_{a, \alpha},
$$

where

$$
k=\frac{T^{\alpha-\frac{1}{2}}}{\Gamma(\alpha) \sqrt{2 \alpha-1}} .
$$


Now, we put

$$
\begin{aligned}
& C_{1}:=\frac{1}{2}\left(1-L T k^{2}\right), \\
& C_{2}:=\frac{1}{2}\left(1+L T k^{2}\right) .
\end{aligned}
$$

We suppose that the Lipschitz constant $L>0$ of the function $h$ satisfies the condition $L T k^{2}<1$.

Definition 2.3 A function

$$
u \in\left\{u \in \operatorname{AC}([0, T]): \int_{t_{j}}^{t_{j+1}}\left(\left|{ }_{0}^{c} D_{t}^{\alpha} u(t)\right|^{2}+|u(t)|^{2}\right) \mathrm{d} t<\infty, j=0, \ldots n\right\}
$$

is said to be a classical solution of problem $\left(D_{\lambda, \mu}\right)$ if

$$
{ }_{t} D_{T}^{\alpha}\left({ }_{0}^{c} D_{t}^{\alpha} u(t)\right)+a(t) u(t)=\lambda f(t, u(t))+h(u(t)), \quad \text { a.e. } t \in[0, T] \backslash\left\{t_{1}, \ldots, t_{n}\right\},
$$

the limits ${ }_{t} D_{T}^{\alpha-1}\left({ }_{0}^{c} D_{t}^{\alpha} u\right)\left(t_{j}^{+}\right)$and ${ }_{t} D_{T}^{\alpha-1}\left({ }_{0}^{c} D_{t}^{\alpha} u\right)\left(t_{j}^{-}\right)$exist, $\Delta\left({ }_{t} D_{T}^{\alpha-1}\left({ }_{0}^{c} D_{t}^{\alpha} u\right)\right)\left(t_{j}\right)=\mu I_{j}\left(u\left(t_{j}\right)\right)$ and $u(0)=u(T)=0$.

We have the following definition of a weak solution for the problem $\left(D_{\lambda, \mu}\right)$.

Definition 2.4 A function $u \in \mathrm{E}^{\alpha}$ is said to be a weak solution of the problem $\left(D_{\lambda, \mu}\right)$, if for every $v \in \mathrm{E}^{\alpha}$,

$$
\begin{gathered}
\int_{0}^{T}\left[\left({ }_{0}^{c} D_{t}^{\alpha} u(t)\right)\left({ }_{0}^{c} D_{t}^{\alpha} v(t)\right)+a(t) u(t) v(t)\right] \mathrm{d} t+\mu \sum_{j=1}^{n} I_{j}\left(u\left(t_{j}\right)\right) v\left(t_{j}\right) \\
\quad=\lambda \int_{0}^{T} f(t, u(t)) v(t) \mathrm{d} t+\int_{0}^{T} h(u(t)) v(t) \mathrm{d} t .
\end{gathered}
$$

Lemma 2.3 ([33], Lemma 2.1) The function $u \in \mathrm{E}^{\alpha}$ is a weak solution of $\left(D_{\lambda, \mu}\right)$ if and only if $u$ is a classical solution of $\left(D_{\lambda, \mu}\right)$.

Our basic tool to guarantee the existence of infinitely many classical solutions for the problem $\left(D_{\lambda, \mu}\right)$ is a smooth version of Theorem 2.1 of [47], which is a more precise version of Ricceri's variational principle [48], which we recall here. This result has relevance for the celebrated three critical points theorem of Pucci and Serrin $[55,56]$.

Theorem 2.4 Let $X$ be a reflexive real Banach space, let $\Phi, \Psi: X \rightarrow \mathbb{R}$ be two Gâteaux differentiable functionals such that $\Phi$ is sequentially weakly lower semi-continuous, strongly continuous, and coercive, and $\Psi$ is sequentially weakly upper semi-continuous. For every $r>\inf _{X} \Phi$, let us put

$$
\varphi(r):=\inf _{u \in \Phi^{-1}(-\infty, r)} \frac{\sup _{v \in \Phi^{-1}(-\infty, r)} \Psi(v)-\Psi(u)}{r-\Phi(u)}
$$


and

$$
\gamma:=\liminf _{r \rightarrow+\infty} \varphi(r), \quad \delta:=\liminf _{\left.r \rightarrow \inf _{X} \Phi\right)^{+}} \varphi(r) .
$$

Then one has:

(a) For every $r>\inf _{X} \Phi$ and every $\left.\lambda \in\right] 0, \frac{1}{\varphi(r)}$ [, the restriction of the functional $I_{\lambda}=\Phi-\lambda \Psi$ to $\Phi^{-1}(]-\infty, r[)$ admits a global minimum, which is a critical point (local minimum) of $I_{\lambda}$ in $X$.

(b) If $\gamma<+\infty$ then, for each $\lambda \in] 0, \frac{1}{\gamma}[$, the following alternative holds: either

(b $)_{1} I_{\lambda}$ possesses a global minimum, or

$\left(b_{2}\right)$ there is a sequence $\left\{u_{n}\right\}$ of critical points (local minima) of $I_{\lambda}$ such that

$$
\lim _{n \rightarrow+\infty} \Phi\left(u_{n}\right)=+\infty
$$

(c) If $\delta<+\infty$ then, for each $\lambda \in] 0, \frac{1}{\delta}[$, the following alternative holds: either

$\left(c_{1}\right)$ there is a global minimum of $\Phi$ which is a local minimum of $I_{\lambda}$, or

$\left(\mathrm{c}_{2}\right)$ there is a sequence of pairwise distinct critical points (local minima) of $I_{\lambda}$ which weakly converges to a global minimum of $\Phi$.

Corresponding to the functions $f, h$, and $I_{j}, j=1, \ldots, n$, we introduce the functions $F$ : $[0, T] \times \mathbb{R} \longrightarrow \mathbb{R}, H: \mathbb{R} \rightarrow \mathbb{R}$, and $J_{j}:[0, T] \times \mathbb{R} \rightarrow \mathbb{R}, j=1, \ldots, n$, respectively, as follows:

$$
\begin{aligned}
& F(t, \xi):=\int_{0}^{\xi} f(t, x) \mathrm{d} x, \quad \text { for all } \xi \in \mathbb{R}, \\
& H(\xi):=\int_{0}^{\xi} h(x) \mathrm{d} x, \quad \text { for all } \xi \in \mathbb{R}
\end{aligned}
$$

and

$$
J_{j}(x)=\int_{0}^{x} I_{j}(\xi) \mathrm{d} \xi, \quad j=1, \ldots, n, \text { for every } x \in \mathbb{R}
$$

A specific case of our main result is the following theorem.

Theorem 2.5 Let $\alpha \in(1 / 2,1], t_{1} \in(0,1)$, and let $f: \mathbb{R} \rightarrow \mathbb{R}$ be a non-negative continuous function and put $F(x)=\int_{0}^{x} f(\xi) \mathrm{d} \xi$ for all $x \in \mathbb{R}$. Assume that

$$
\liminf _{\xi \rightarrow+\infty} \frac{F(\xi)}{\xi^{2}}=0 \quad \text { and } \quad \limsup _{\xi \rightarrow+\infty} \frac{F(\xi)}{\xi^{2}}=+\infty
$$

Then, for every continuous function $I: \mathbb{R} \rightarrow \mathbb{R}$ whose $J(x)=\int_{0}^{x} I(\xi) \mathrm{d} \xi$ for every $x \in \mathbb{R}$, is a non-positive function and satisfying the condition

$$
J_{\star}:=\lim _{\xi \rightarrow \infty} \frac{\sup _{|x| \leq \xi}(-J(x))}{\xi^{2}}<+\infty,
$$


and for every $\mu \in\left[0, \mu_{\star}\left[\right.\right.$ where $\mu_{\star}:=\frac{C_{1}}{k^{2} J_{\star}}\left(1-\frac{k^{2}}{C_{1}} \liminf _{\xi \rightarrow+\infty} \frac{F(\xi)}{\xi^{2}}\right)$, the problem

$$
\begin{aligned}
& { }_{t} D_{T}^{\alpha}\left({ }_{0}^{c} D_{t}^{\alpha} u(t)\right)+u(t)=f(u(t))+h(u(t)), \quad t \neq t_{1}, \text { a.e. } t \in[0,1], \\
& \Delta\left({ }_{t} D_{T}^{\alpha-1}\left({ }_{0}^{c} D_{t}^{\alpha} u\right)\right)\left(t_{1}\right)=\mu I\left(u\left(t_{1}\right)\right), \\
& u(0)=u(1)=0,
\end{aligned}
$$

has an unbounded sequence of classical solutions.

\section{Main results}

First we set

$$
A(\alpha):=\frac{1}{\Gamma^{2}(1-\alpha)}\left(\frac{T}{4}\right)^{1-2 \alpha} \frac{6 \alpha^{2}-19 \alpha+16}{(1-\alpha)^{2}(2-\alpha)(3-2 \alpha)} .
$$

Now we formulate our main result as follows.

\section{Theorem 3.1 Assume that}

(A1) $F(t, x) \geq 0$ for all $t \in\left[0, \frac{T}{4}\right] \cup\left[\frac{3 T}{4}, T\right]$ and $x \in \mathbb{R}$;

(A2) $\liminf _{\xi \rightarrow+\infty} \frac{\int_{0}^{T} \sup _{|x| \leq \xi} F(t, x) \mathrm{d} t}{\xi^{2}}<\frac{C_{1}}{\left(A(\alpha)+\frac{2 T|\|| \mid \infty}{3}\right) k^{2} C_{2}} \lim \sup _{\xi \rightarrow+\infty} \frac{\int_{\frac{T}{4}}^{\frac{3 T}{4}} F(t, \xi) \mathrm{d} t}{\xi^{2}}$.

Then, for each $\lambda \in] \lambda_{1}, \lambda_{2}$ [ where

$$
\lambda_{1}:=\frac{\left(A(\alpha)+\frac{2 T\|a\|_{\infty}}{3}\right) C_{2}}{\lim \sup _{\xi \rightarrow+\infty} \frac{\int_{\frac{T}{4}}^{\frac{3 T}{4}} F(t, \xi) \mathrm{d} t}{\xi^{2}}}
$$

and

$$
\lambda_{2}:=\frac{C_{1}}{k^{2} \liminf _{\xi \rightarrow+\infty} \frac{\int_{0}^{T} \sup _{|x| \leq \xi} F(t, x) \mathrm{d} t}{\xi^{2}}},
$$

for all continuous functions $I_{j}: \mathbb{R} \longrightarrow \mathbb{R}, j=1, \ldots, n$, for which $J_{j}(x)=\int_{0}^{x} I_{j}(\xi) \mathrm{d} \xi, j=1, \ldots, n$, for every $x \in \mathbb{R}$, are non-positive functions and satisfying the condition

$$
J_{\infty}:=\lim _{\xi \rightarrow \infty} \frac{\sup _{|x| \leq \xi} \sum_{j=1}^{n}-J_{j}(x)}{\xi^{2}}<\infty
$$

and for every $\mu \in\left[0, \mu_{J, \lambda}\left[\right.\right.$ where $\mu_{J, \lambda}:=\frac{C_{1}}{T k^{2} J_{\infty}}\left(1-\frac{\lambda}{\lambda_{2}}\right)$, the problem $\left(D_{\lambda, \mu}\right)$ has an unbounded sequence of classical solutions.

Proof Fix $\bar{\lambda} \in] \lambda_{1}, \lambda_{2}\left[\right.$ and assume that $I_{j}, j=1, \ldots, n$, are the functions satisfying the condition (4). Since $\bar{\lambda}<\lambda_{2}$, one has $\mu_{J, \bar{\lambda}}>0$. Fix $\bar{\mu} \in\left[0, \mu_{J, \bar{\lambda}}\left[\right.\right.$ and put $v_{1}:=\lambda_{1}$ and $v_{2}:=$ $\frac{C_{1} \lambda_{2}}{C_{1}+\frac{\bar{\mu}}{\bar{\lambda}} \lambda_{2} T k^{2} J_{\infty}}$. If $J_{\infty}=0$, clearly, $v_{1}=\lambda_{1}$ and $v_{2}=\lambda_{2}$, and $\left.\bar{\lambda} \in\right] \nu_{1}, \nu_{2}\left[\right.$. If $J_{\infty} \neq 0$, since $\bar{\mu}<\mu_{J, \bar{\lambda}}$, we obtain $\frac{\bar{\lambda}}{\lambda_{2}}+\frac{\bar{\mu} T k^{2} J_{\infty}}{C_{1}}<1$, and so $\frac{C_{1} \lambda_{2}}{C_{1}+\frac{\bar{\mu}}{\lambda} \lambda_{2} T k^{2} J_{\infty}}>\bar{\lambda}$, namely, $\bar{\lambda}<v_{2}$. Hence, since $\bar{\lambda}>\lambda_{1}=v_{1}$, one has $\bar{\lambda} \in] v_{1}, v_{2}\left[\right.$. Now, set $Q(t, x)=F(t, x)-\frac{\bar{\mu}}{\bar{\lambda}} \sum_{j=1}^{n} J_{j}(x)$ for all $(t, x) \in[0, T] \times \mathbb{R}$. Take 
$X=\mathrm{E}^{\alpha}$ and define on $X$ two functionals $\Phi$ and $\Psi$ by setting, for each $u \in X$,

$$
\Phi(u):=\frac{1}{2}\|u\|_{a, \alpha}^{2}-\int_{0}^{T} H(u(t)) \mathrm{d} t
$$

and

$$
\Psi(u)=\int_{0}^{T} F(t, u(t)) \mathrm{d} t-\frac{\bar{\mu}}{\bar{\lambda}} \sum_{j=1}^{n} J_{j}\left(u\left(t_{j}\right)\right) .
$$

It is clear that $\Psi$ is a Gâteaux differentiable functional, sequentially weakly upper semicontinuous, whose Gâteaux derivative at the point $u \in X$ is the functional $\Psi^{\prime}(u) \in X^{*}$, given by

$$
\Psi^{\prime}(u) v=\int_{0}^{T} f(t, u(t)) v(t) \mathrm{d} t-\frac{\bar{\mu}}{\bar{\lambda}} \sum_{j=1}^{n} I_{j}\left(u\left(t_{j}\right)\right) v\left(t_{j}\right)
$$

for every $v \in X$, and $\Psi^{\prime}: X \rightarrow X^{*}$ is a compact operator (see [33]). Moreover, $\Phi$ is a Gâteaux differentiable functional of which the Gâteaux derivative at the point $u \in X$ is the functional $\Phi^{\prime}(u) \in X^{*}$, given by

$$
\Phi^{\prime}(u) v=\int_{0}^{T}\left[\left({ }_{0}^{c} D_{t}^{\alpha} u(t)\right)\left({ }_{0}^{c} D_{t}^{\alpha} v(t)\right)+a(t) u(t) v(t)\right] \mathrm{d} t-\int_{0}^{T} h(u(t)) v(t) \mathrm{d} t
$$

for every $v \in X$. Moreover, by the sequentially weakly lower semi-continuity of $\|u\|_{a, \alpha}$ and the continuity of $H, \Phi$ is sequentially weakly lower semi-continuous in $X$. Now from the facts $-L|\xi| \leq h(\xi) \leq L|\xi|$ for every $\xi \in \mathbb{R}$, and taking (2) and (3) into account, for every $u \in X$ we have

$$
C_{1}\|u\|_{a, \alpha}^{2} \leq \Phi(u) \leq C_{2}\|u\|_{a, \alpha}^{2} .
$$

Put $I_{\bar{\lambda}}:=\Phi-\bar{\lambda} \Psi$. Now, we are to demonstrate that $\gamma<+\infty$, where $\gamma$ has been defined in Theorem 2.4. Let $\left\{\xi_{n}\right\}$ be a real sequence such that $\xi_{n}>0$ for all $n \in \mathbb{N}$ and $\xi_{n} \rightarrow+\infty$ as $n \rightarrow \infty$ and

$$
\lim _{n \rightarrow \infty} \frac{\int_{0}^{T} \sup _{|x| \leq \xi_{n}} Q(t, x) \mathrm{d} t}{\xi_{n}^{2}}=\liminf _{\xi \rightarrow+\infty} \frac{\int_{0}^{T} \sup _{|x| \leq \xi} Q(t, x) \mathrm{d} t}{\xi^{2}} .
$$

Put $r_{n}=\frac{C_{1} \xi_{n}^{2}}{k^{2}}$ for all $n \in \mathbb{N}$. Since $\xi_{n}>0, r_{n}>0$ for all $n \in \mathbb{N}$. Now let $u \in \Phi^{-1}\left(-\infty, r_{n}\right)$, owing to (5), we have

$$
C_{1}\|u\|_{a, \alpha}^{2} \leq \Phi(u)<r_{n}
$$

By (2) and (6) we have $\|u\|_{\infty} \leq \xi_{n}$. Thus

$$
\Phi^{-1}\left(-\infty, r_{n}\right) \subseteq\left\{u:\|u\|_{\infty} \leq \xi_{n}\right\}
$$


Hence, since $\Phi(0)=\Psi(0)=0$, for every $n$ large enough, one has

$$
\begin{aligned}
\varphi\left(r_{n}\right) & =\inf _{u \in \Phi^{-1}\left(-\infty, r_{n}\right)} \frac{\left(\sup _{v \in \Phi^{-1}\left(-\infty, r_{n}\right)} \Psi(v)\right)-\Psi(u)}{r_{n}-\Phi(u)} \leq \frac{\sup _{v \in \Phi^{-1}\left(-\infty, r_{n}\right)} \Psi(v)}{r_{n}} \\
& \leq \frac{\int_{0}^{T} \sup _{|x| \leq \xi_{n}} Q(t, x) \mathrm{d} t}{\frac{C_{1} \xi_{n}^{2}}{k^{2}}}=\frac{\int_{0}^{T} \sup _{|x| \leq \xi_{n}}\left(F(t, x)-\frac{\bar{\mu}}{\bar{\lambda}} \sum_{j=1}^{n} J_{j}(x)\right) \mathrm{d} t}{\frac{C_{1} \xi_{n}^{2}}{k^{2}}} \\
& \leq \frac{\int_{0}^{T} \sup _{|x| \leq \xi_{n}} F(t, x) \mathrm{d} t}{\frac{C_{1} \xi_{n}^{2}}{k^{2}}}+\frac{\bar{\mu}}{\bar{\lambda}} \frac{T \sup _{|x| \leq \xi_{n}} \sum_{j=1}^{n}-J_{j}(x)}{\frac{C_{1} \xi_{n}^{2}}{k^{2}}} .
\end{aligned}
$$

Moreover, by Assumption (A2) one has

$$
\liminf _{\xi \rightarrow+\infty} \frac{\int_{0}^{T} \sup _{|x| \leq \xi} F(t, x) \mathrm{d} t}{\frac{C_{1} \xi^{2}}{k^{2}}}<+\infty
$$

which implies

$$
\lim _{n \rightarrow \infty} \frac{\int_{0}^{T} \sup _{|x| \leq \xi_{n}} F(t, x) \mathrm{d} t}{\frac{C_{1} \xi_{n}^{2}}{k^{2}}}<+\infty
$$

Then, regarding (4) and (7), we have

$$
\lim _{n \rightarrow \infty} \frac{\int_{0}^{T} \sup _{|x| \leq \xi_{n}} F(t, x) \mathrm{d} t}{\frac{C_{1} \xi_{n}^{2}}{k^{2}}}+\lim _{n \rightarrow \infty} \frac{\bar{\mu}}{\bar{\lambda}} \frac{T \sup _{|x| \leq \xi_{n}} \sum_{j=1}^{n}-J_{j}(x)}{\frac{C_{1} \xi_{n}^{2}}{k^{2}}}<+\infty
$$

from which follows

$$
\lim _{n \rightarrow \infty} \frac{\int_{0}^{T} \sup _{|x| \leq \xi_{n}}\left(F(t, x)-\frac{\bar{\mu}}{\bar{\lambda}} \sum_{j=1}^{n} J_{j}(x)\right) \mathrm{d} t}{\frac{C_{1} \xi_{n}^{2}}{k^{2}}}<+\infty
$$

Therefore,

$$
\gamma \leq \liminf _{n \rightarrow+\infty} \varphi\left(r_{n}\right) \leq \lim _{n \rightarrow \infty} \frac{\int_{0}^{T} \sup _{|x| \leq \xi_{n}}\left(F(t, x)-\frac{\bar{\mu}}{\bar{\lambda}} \sum_{j=1}^{n} J_{j}(x)\right) \mathrm{d} t}{\frac{C_{1} \xi_{n}^{2}}{k^{2}}}<+\infty
$$

Since

$$
\frac{\int_{0}^{T} \sup _{|x| \leq \xi_{n}} Q(t, x) \mathrm{d} t}{\xi_{n}^{2}} \leq \frac{\int_{0}^{T} \sup _{|x| \leq \xi_{n}} F(t, x) \mathrm{d} t}{\xi_{n}^{2}}+\frac{\bar{\mu}}{\bar{\lambda}} \frac{T \sup _{|x| \leq \xi_{n}} \sum_{j=1}^{n}-J_{j}(x)}{\xi_{n}^{2}},
$$

taking (4) into account, one has

$$
\liminf _{\xi \rightarrow+\infty} \frac{\int_{0}^{T} \sup _{|x| \leq \xi} Q(t, x) \mathrm{d} t}{\xi^{2}} \leq \liminf _{\xi \rightarrow+\infty} \frac{\int_{0}^{T} \sup _{|x| \leq \xi} F(t, x) \mathrm{d} t}{\xi^{2}}+\frac{\bar{\mu} T}{\bar{\lambda}} J_{\infty} .
$$


Moreover, $J_{j}, j=1, \ldots, n$, are non-positive, we have

$$
\limsup _{|\xi| \rightarrow+\infty} \frac{\int_{\frac{T}{4}}^{\frac{3 T}{4}} Q(t, \xi) \mathrm{d} t}{\xi^{2}} \geq \limsup _{|\xi| \rightarrow+\infty} \frac{\int_{\frac{T}{4}}^{\frac{3 T}{4}} F(t, \xi) \mathrm{d} t}{\xi^{2}} .
$$

Therefore, from (9) and (10), and from Assumption (A2) and (8), one has

$$
\begin{aligned}
\bar{\lambda} \in] v_{1}, v_{2}[\subseteq] & \frac{\left(A(\alpha)+\frac{2 T\|a\|_{\infty}}{3}\right) C_{2}}{\lim \sup _{|\xi| \rightarrow+\infty} \frac{\int_{\frac{T}{4}}^{\frac{3 T}{4}} F(t, \xi) \mathrm{d} t}{\xi^{2}}}, \frac{C_{1}}{k^{2} \liminf _{\xi \rightarrow+\infty} \frac{\int_{0}^{T} \sup _{|x| \leq \xi} F(t, x) \mathrm{d} t}{\xi^{2}}}[ \\
& \subseteq] 0, \frac{1}{\gamma}[.
\end{aligned}
$$

For the fixed $\bar{\lambda}$, the inequality (8) guarantees that the condition (b) of Theorem 2.4 can be used and either $I_{\bar{\lambda}}$ has a global minimum or there is a series $\left\{u_{n}\right\}$ of weak solutions of the problem $\left(D_{\lambda, \mu}\right)$, so that $\lim _{n \rightarrow \infty}\|u\|_{a, \alpha}=+\infty$.

The other step is to investigate that the functional $I_{\bar{\lambda}}$ has no global minimum. Since

$$
\frac{1}{\bar{\lambda}}<\limsup _{|\xi| \rightarrow+\infty} \frac{\int_{\frac{T}{4}}^{\frac{3 T}{4}} F(t, \xi) \mathrm{d} t}{\left(A(\alpha)+\frac{2 T\|a\|_{\infty}}{3}\right) C_{2} \xi^{2}}
$$

we can consider a real sequence $\left\{\gamma_{n}\right\}$ and a positive constant $\tau$ so that $\gamma_{n} \rightarrow+\infty$ as $n \rightarrow \infty$ and

$$
\frac{1}{\bar{\lambda}}<\tau<\frac{\int_{\frac{T}{4}}^{\frac{T}{4}} F\left(t, \gamma_{n}\right) \mathrm{d} t}{\left(A(\alpha)+\frac{2 T\|a\|_{\infty}}{3}\right) C_{2} \gamma_{n}^{2}}
$$

for each $n \in \mathbb{N}$ large enough. Let $\left\{w_{n}\right\}$ be a sequence in $X$ defined by

$$
w_{n}(t)= \begin{cases}\frac{4 \gamma_{n}}{T} t, & \text { if } t \in\left[0, \frac{T}{4}\right), \\ \gamma_{n}, & \text { if } t \in\left[\frac{T}{4}, \frac{3 T}{4}\right], \\ \frac{4 \gamma_{n}}{T}(T-t), & \text { if } t \in\left(\frac{3 T}{4}, T\right] .\end{cases}
$$

Obviously, one has

$$
w_{n}^{\prime}(t)= \begin{cases}\frac{4 \gamma_{n}}{T}, & \text { if } t \in\left(0, \frac{T}{4}\right), \\ 0, & \text { if } t \in\left(\frac{T}{4}, \frac{3 T}{4}\right), \\ -\frac{4 \gamma_{n}}{T}, & \text { if } t \in\left(\frac{3 T}{4}, T\right),\end{cases}
$$

and

$$
\begin{array}{rlrl}
\left|{ }_{0}^{c} D_{t}^{\alpha} w(t)\right| & =\frac{1}{\Gamma(1-\alpha)}\left(\int_{0}^{T}(t-s)^{-\alpha} w^{\prime}(s) \mathrm{d} s\right) & \\
& =\frac{1}{\Gamma(1-\alpha)} \begin{cases}\frac{4 \gamma_{n}}{T} \frac{t^{1-\alpha}}{1-\alpha}, & \text { if } t \in\left[0, \frac{T}{4}\right), \\
\frac{4 \gamma_{n}}{T} \frac{\left(\frac{T}{4}\right)^{1-\alpha}}{1-\alpha}, & \text { if } t \in\left[\frac{T}{4}, \frac{3 T}{4}\right], \\
\frac{4 \gamma_{n}}{T} \frac{1}{1-\alpha}\left[\left(\frac{T}{4}\right)^{1-\alpha}-\left(t-\left(\frac{3 T}{4}\right)\right)^{1-\alpha}\right], & \text { if } t \in\left(\frac{3 T}{4}, T\right],\end{cases}
\end{array}
$$


so that

$$
\left\|w_{n}\right\|_{a, \alpha}^{2}=A(\alpha) \gamma_{n}^{2}+\int_{0}^{T} a(t)\left|w_{n}(t)\right|^{2} \mathrm{~d} t \leq\left(A(\alpha)+\frac{2 T\|a\|_{\infty}}{3}\right) \gamma_{n}^{2},
$$

and particularly, considering (5), it follows that

$$
\Phi\left(w_{n}\right) \leq\left(A(\alpha)+\frac{2 T\|a\|_{\infty}}{3}\right) C_{2} \gamma_{n}^{2} .
$$

On the other hand, based on the non-positivity of $J_{j}, j=1, \ldots, n$, we observe that

$$
\Psi\left(w_{n}\right) \geq \int_{\frac{T}{4}}^{\frac{3 T}{4}} F\left(t, \gamma_{n}\right) \mathrm{d} t
$$

So, from (11), (13), and (14) we have

$$
\begin{aligned}
I_{\bar{\lambda}}\left(w_{n}\right) & =\Phi\left(w_{n}\right)-\bar{\lambda} \Psi\left(w_{n}\right) \leq\left(A(\alpha)+\frac{2 T\|a\|_{\infty}}{3}\right) C_{2} \gamma_{n}^{2}-\bar{\lambda}\left(\int_{\frac{T}{4}}^{\frac{3 T}{4}} F\left(t, \gamma_{n}\right) \mathrm{d} t\right) \\
& <(1-\bar{\lambda} \tau)\left(A(\alpha)+\frac{2 T\|a\|_{\infty}}{3}\right) C_{2} \gamma_{n}^{2},
\end{aligned}
$$

for every $n \in \mathbb{N}$ large enough. Hence, the functional $I_{\bar{\lambda}}$ is unbounded from below, and it shows that $I_{\bar{\lambda}}$ has no global minimum. Therefore, Theorem 2.4 ensures that there is a sequence $\left\{u_{n}\right\} \subset X$ of critical points of $I_{\bar{\lambda}}$ so that $\lim _{n \rightarrow \infty} \Phi\left(u_{n}\right)=+\infty$, which from (5) shows that $\lim _{n \rightarrow \infty}\left\|u_{n}\right\|_{a, \alpha}=+\infty$. Since the critical points of $I_{\bar{\lambda}}$ are the weak solutions of the problem $\left(D_{\lambda, \mu}\right)$, and thanks to Lemma 2.3 , and considering that they are classical solutions, we have the conclusion.

Remark 3.1 The condition non-positivity of $J_{j}, j=1, \ldots, n$, can be replaced by the following one:

(I) $I_{j}(0)=0$ and $I_{j}(s) s<0$ for all $j=1, \ldots, n$.

In fact, by this assumption for every $j=1, \ldots, n$ we have $J_{j}(x)=\int_{0}^{x} I_{j}(\xi) \mathrm{d} \xi \leq 0$ for $x \in \mathbb{R}$.

Remark 3.2 Under the conditions

$$
\liminf _{\xi \rightarrow+\infty} \frac{\int_{0}^{T} \sup _{|x| \leq \xi} F(t, x) \mathrm{d} t}{\xi^{2}}=0 \text { and } \limsup _{\xi \rightarrow+\infty} \frac{\int_{\frac{T}{4}}^{\frac{3 T}{4}} F(t, \xi) \mathrm{d} t}{\xi^{2}}=\infty,
$$

Theorem 3.1 ensures that for every $\lambda>0$ and for each $\mu \in\left[0, \frac{1}{J_{\infty}}\left[\right.\right.$ the problem $\left(D_{\lambda, \mu}\right)$ admits infinitely many classical solutions. Moreover, if $J_{\infty}=0$, the result holds for every $\lambda>0$ and $\mu \geq 0$.

Now, we give an application of Theorem 3.1.

Example 3.1 Let $\alpha=\frac{5}{9}, T=1, n=1, t_{1}=\frac{1}{2}$, and put

$$
a_{n}:=\frac{2 n !(n+2) !-1}{4(n+1) !}, \quad b_{n}:=\frac{2 n !(n+2) !+1}{4(n+1) !}
$$


for every $n \in \mathbb{N}$. Consider the problem

$$
\begin{aligned}
& { }_{t} D_{1}^{\frac{5}{9}}\left({ }_{0}^{c} D_{t}^{\frac{5}{9}} u(t)\right)+a(t) u(t)=\lambda f(t, u(t))+h(u(t)), \quad t \neq t_{1}, \text { a.e. } t \in[0,1], \\
& \Delta\left({ }_{t} D_{1}^{-\frac{4}{9}}\left({ }_{0}^{c} D_{t}^{\frac{5}{9}} u\right)\right)\left(\frac{1}{2}\right)=\mu I\left(u\left(\frac{1}{2}\right)\right), \\
& u(0)=u(1)=0,
\end{aligned}
$$

where $a(t)=2+\cos t$ for all $t \in[0,1]$,

$$
f(t, x)= \begin{cases}\frac{32 t(n+1) ! !^{2}\left[(n+1) !^{2}-n !^{2}\right]}{\pi} \sqrt{\frac{1}{16(n+1) !^{2}}-\left(x-\frac{n !(n+2)}{2}\right)^{2}} & \text { if }(t, x) \in[0,1] \times \bigcup_{n \in \mathbb{N}}\left[a_{n}, b_{n}\right] \\ 0 & \text { elsewhere }\end{cases}
$$

$h(x)=\frac{\Gamma^{2}\left(\frac{5}{9}\right)}{36} \ln \left(1+x^{2}\right)$, for all $x \in \mathbb{R}$ and $I_{1}(x)=e^{x^{-}}\left(x^{-}\right)\left(2-x^{-}\right)$where $x^{-}=\min \{x, 0\}$, for all $x \in \mathbb{R}$. According to the data above we have $k=3 \Gamma^{-1}\left(\frac{5}{9}\right),\|a\|_{\infty}=3, L=\frac{\Gamma^{2}\left(\frac{5}{9}\right)}{18}, C_{1}=0.25$, $C_{2}=0.75, A\left(\frac{5}{9}\right)=11.08125 \sqrt[9]{4} \Gamma^{-2}\left(\frac{4}{9}\right)$, and $\int_{n !}^{(n+1) !} f(1, x) \mathrm{d} x=(n+1) !^{2}-n !^{2}$ for every $n \in \mathbb{N}$. Then one has $\lim _{n \rightarrow+\infty} \frac{F\left(1, a_{n}\right)}{a_{n}^{2}}=0$ and $\lim _{n \rightarrow+\infty} \frac{F\left(1, b_{n}\right)}{b_{n}^{2}}=4$. So, $\liminf _{\xi \rightarrow \infty} \frac{F(1, \xi)}{\xi^{2}}=0$ and $\lim \sup _{\xi \rightarrow \infty} \frac{F(1, \xi)}{\xi^{2}}=4$. Therefore,

$$
\liminf _{\xi \rightarrow+\infty} \frac{\int_{0}^{1} \sup _{|x| \leq \xi} F(t, x) \mathrm{d} t}{|\xi|^{2}}=\liminf _{\xi \rightarrow+\infty} \frac{\int_{0}^{1} t \sup _{|x| \leq \xi} F(1, x) \mathrm{d} t}{|\xi|^{2}}=0
$$

and

$$
\begin{aligned}
& \frac{C_{1}}{\left(A\left(\frac{5}{9}\right)+\frac{2 T\|a\|_{\infty}}{3}\right) k^{2} C_{2}} \limsup _{\xi \rightarrow+\infty} \frac{\int_{\frac{1}{4}}^{\frac{3}{4}} F(t, \xi) \mathrm{d} t}{|\xi|^{2}} \\
& =\frac{\Gamma^{2}\left(\frac{5}{9}\right)}{54\left(2+11.08125 \sqrt[9]{2} \Gamma^{-2}\left(\frac{4}{9}\right)\right)} \limsup _{\xi \rightarrow+\infty} \frac{F(1, \xi)}{|\xi|^{2}} \\
& =\frac{2 \Gamma^{2}\left(\frac{5}{9}\right)}{27\left(2+11.08125 \sqrt[9]{2} \Gamma^{-2}\left(\frac{4}{9}\right)\right)} .
\end{aligned}
$$

Hence, using Theorem 3.1, since

$$
J_{\infty}:=\lim _{\xi \rightarrow \infty} \frac{\sup _{|x| \leq \xi}\left(-J_{1}(x)\right)}{\xi^{2}}=0<\infty
$$

the problem (15) for every $\lambda>\frac{27\left(2+11.08125 \sqrt[9]{2} \Gamma^{-2}\left(\frac{4}{9}\right)\right)}{2 \Gamma^{2}\left(\frac{5}{9}\right)}$ and $\mu \in[0,+\infty)$ has an unbounded sequence of classical solutions.

Remark 3.3 The following condition:

(A'2) there exist two sequence $\left\{\theta_{n}\right\}$ and $\left\{\eta_{n}\right\}$ with $\eta_{n}>0$ for every $n \in \mathbb{N}$ and

$$
\left(A(\alpha)+\frac{2 T\|a\|_{\infty}}{3}\right) C_{2} \theta_{n}^{2}<\frac{C_{1}}{k^{2}} \eta_{n}^{2}
$$


for all $n \in \mathbb{N}$ and $\lim _{n \rightarrow+\infty} \eta_{n}=+\infty$ such that

$$
\lim _{n \rightarrow+\infty} \frac{\int_{0}^{T} \sup _{|x| \leq \eta_{n}} F(t, x) \mathrm{d} t-\int_{\frac{T}{4}}^{\frac{3 T}{4}} F\left(t, \theta_{n}\right) \mathrm{d} t}{\frac{C_{1}}{k^{2}} \eta_{n}^{2}-\left(A(\alpha)+\frac{2 T\|a\|_{\infty}}{3}\right) C_{2} \theta_{n}^{2}}<\limsup _{|\xi| \rightarrow+\infty} \frac{\int_{\frac{T}{4}}^{\frac{3 T}{4}} F(t, \xi) \mathrm{d} t}{\left(A(\alpha)+\frac{2 T\|a\| \infty}{3}\right) C_{2} \xi^{2}}
$$

is more general than condition (A2) of Theorem 3.1. In fact, by choosing $\theta_{n}=0$ for all $n \in \mathbb{N}$ from (A'2) we obtain (A2). If we assume (A'2) instead of (A2) and choose $r_{n}=\frac{C_{1} \eta_{n}^{2}}{k^{2}}$ for all $n \in \mathbb{N}$, by the same arguing as for Theorem 3.1, we obtain

$$
\begin{aligned}
\varphi\left(r_{n}\right) & \leq \frac{\sup _{\left.\left.v \in \Phi^{-1}(]-\infty, r_{n}\right]\right)} \Psi(v)-\int_{0}^{T} F\left(t, w_{n}(t)\right) \mathrm{d} t}{r_{n}-\frac{1}{2}\left\|w_{n}\right\|_{a, \alpha}^{2}+\int_{0}^{T} H\left(w_{n}(t)\right) \mathrm{d} t} \\
& \leq \frac{\int_{0}^{t} \sup _{|x| \leq \eta_{n}} F(t, x) \mathrm{d} t-\int_{\frac{T}{4}}^{\frac{3 T}{4}} F\left(t, \theta_{n}\right) \mathrm{d} t}{\frac{C_{1}}{k^{2}} \eta_{n}^{2}-\left(A(\alpha)+\frac{2 T\|a\|_{\infty}}{3}\right) C_{2} \theta_{n}^{2}},
\end{aligned}
$$

where $w_{n}(t)$ is the same as (12) but $\gamma_{n}$ replaced by $\theta_{n}$, and we have the same conclusion as in Theorem 3.1 with the interval $] \lambda_{1}, \lambda_{2}$ [ replaced by the interval

$$
\left.\Lambda^{\prime}=\right] \frac{1}{\lim \sup _{|\xi| \rightarrow+\infty} \frac{\int_{\frac{T}{4}}^{\frac{3 T}{4}} F(t, \xi) \mathrm{d} t}{\left(A(\alpha)+\frac{\left.2 T|a|\right|_{\infty}}{3}\right) C_{2} \xi^{2}}}, \frac{1}{k^{2} \lim _{n \rightarrow+\infty} \frac{\int_{0}^{T} \sup _{|x| \leq \eta_{n}} F(t, x) \mathrm{d} t-\int_{\frac{T}{4}}^{\frac{3 T}{4}} F\left(t, \theta_{n}\right) \mathrm{d} t}{C_{1} \eta_{n}^{2}-\left(A(\alpha)+\frac{2 T \| a||_{\infty}}{3}\right) k^{2} C_{2} \theta_{n}^{2}}}[.
$$

Here, we point out a simple consequence of Theorem 3.1.

Corollary 3.2 Assume that Assumption (A1) holds. Furthermore, suppose that

(B1) $\liminf _{\xi \rightarrow+\infty} \frac{\int_{0}^{T} \sup _{|x| \leq \xi} F(t, x) \mathrm{d} t}{\xi^{2}}<\frac{C_{1}}{k^{2}}$;

(B2) $\lim \sup _{\xi \rightarrow+\infty} \frac{\int_{\frac{T}{4}}^{\frac{T}{4}} F(t, \xi) \mathrm{d} t}{\xi^{2}}>\left(A(\alpha)+\frac{2 T\|a\|_{\infty}}{3}\right) C_{2}$,

for all continuous functions $I_{j}: \mathbb{R} \longrightarrow \mathbb{R}, j=1, \ldots, n$, for which $J_{j}(x)=\int_{0}^{x} I_{j}(\xi) \mathrm{d} \xi, j=1, \ldots, n$, for every $x \in \mathbb{R}$ are non-positive functions and satisfy the condition (4), and for every $\mu \in$ $\left[0, \mu_{J, 1}[\right.$ where

$$
\mu_{J, 1}:=\frac{C_{1}}{T k^{2} J_{\infty}}\left(1-\frac{k^{2}}{C_{1}} \liminf _{\xi \rightarrow+\infty} \frac{\int_{0}^{T} \sup _{|x| \leq \xi} F(t, x) \mathrm{d} t}{\xi^{2}}\right),
$$

the problem

$$
\begin{aligned}
& { }_{t} D_{T}^{\alpha}\left({ }_{0}^{c} D_{t}^{\alpha} u(t)\right)+a(t) u(t)=f(t, u(t))+h(u(t)), \quad t \neq t_{j}, \text { a.e. } t \in[0, T], \\
& \Delta\left({ }_{t} D_{T}^{\alpha-1}\left({ }_{0}^{c} D_{t}^{\alpha} u\right)\right)\left(t_{j}\right)=\mu I_{j}\left(u\left(t_{j}\right)\right), \quad j=1, \ldots n, \\
& u(0)=u(T)=0,
\end{aligned}
$$

has an unbounded sequence of classical solutions.

Remark 3.4 Theorem 2.5 is an immediately consequence of Corollary 3.2 when $\mu=0$.

We here give the following consequence of the main result. 
Corollary 3.3 Let $f_{1}:[0, T] \longrightarrow \mathbb{R}$ be a non-negative continuous function and let $F_{1}(x)=$ $\int_{0}^{x} f_{1}(\xi) \mathrm{d} \xi$ for all $x \in \mathbb{R}$. Assume that

(D1) $\liminf _{\xi \rightarrow+\infty} \frac{F_{1}(\xi)}{\xi^{2}}<+\infty$;

(D2) $\lim \sup _{\xi \rightarrow+\infty} \frac{F_{1}(\xi)}{\xi^{2}}=+\infty$.

Then, for every $b_{i} \in \mathrm{L}^{1}([0, T])$ for $1 \leq i \leq n$, with $\min _{x \in[0, T]}\left\{b_{i}(x), 1 \leq i \leq n\right\}>0$ and with $b_{1} \neq 0$, and for any non-negative continuous functions $f_{i}: \mathbb{R} \rightarrow \mathbb{R}$ with $F_{i}(x)=\int_{0}^{x} f_{i}(\xi) \mathrm{d} \xi$ for all $x \in \mathbb{R}$ for $2 \leq i \leq n$, satisfying

$$
\max \left\{\sup _{\xi \in \mathbb{R}} F_{i}(\xi) ; 2 \leq i \leq n\right\} \leq 0
$$

and

$$
\min \left\{\liminf _{\xi \rightarrow+\infty} \frac{F_{i}(\xi)}{\xi^{2}} ; 2 \leq i \leq n\right\}>-\infty
$$

for each

$$
\lambda \in] 0, \frac{C_{1}}{k^{2} \liminf _{\xi \rightarrow+\infty} \frac{F_{1}(\xi)}{\xi^{2}}}[,
$$

for all continuous function $I_{j}: \mathbb{R} \longrightarrow \mathbb{R}, 1 \leq j \leq n$, for which $J_{j}(x)=\int_{0}^{x} I_{j}(\xi) \mathrm{d} \xi, 1 \leq j \leq n$, for every $x \in \mathbb{R}$ are non-positive functions and satisfy the condition (4), and for every $\mu \in$ $\left[0, \mu_{J, \lambda}[\right.$ where

$$
\mu_{J, \lambda}:=\frac{C_{1}}{T k^{2} J_{\infty}}\left(1-\frac{\lambda T k^{2}}{C_{1}} \liminf _{\xi \rightarrow+\infty} \frac{F_{1}(\xi)}{\xi^{2}}\right)
$$

the problem

$$
\begin{aligned}
& { }_{t} D_{T}^{\alpha}\left({ }_{0}^{c} D_{t}^{\alpha} u(t)\right)+a(t) u(t)=\lambda \sum_{i=1}^{n} b_{i}(t) f_{i}(u(t))+h(u(t)), \quad t \neq t_{j}, \text { a.e. } t \in[0, T], \\
& \Delta\left({ }_{t} D_{T}^{\alpha-1}\left({ }_{0}^{c} D_{t}^{\alpha} u\right)\right)\left(t_{j}\right)=\mu I_{j}\left(u\left(t_{j}\right)\right), \quad j=1, \ldots, n, \\
& u(0)=u(T)=0,
\end{aligned}
$$

has an unbounded sequence of classical solutions.

Proof Set $F(\xi)=\sum_{i=1}^{n} b(t) F_{i}(\xi)$ for all $\xi \in \mathbb{R}$. Assumption $(D 2)$ along with the condition

$$
\min \left\{\liminf _{\xi \rightarrow \mathbb{R}} \frac{F_{i}(\xi)}{\xi^{2}} ; 2 \leq i \leq n\right\}>-\infty
$$

ensures

$$
\limsup _{\xi \rightarrow+\infty} \frac{\int_{\frac{T}{4}}^{\frac{3 T}{4}} F(t, \xi) \mathrm{d} t}{\xi^{2}}=\limsup _{\xi \rightarrow+\infty} \frac{\sum_{i=1}^{n} F_{i}(\xi) \int_{\frac{T}{4}}^{\frac{3 T}{4}} b_{i}(t) \mathrm{d} t}{\xi^{2}}=+\infty .
$$

Moreover, from the assumption (D1) and the condition

$$
\max \left\{\sup _{\xi \in \mathbb{R}} F_{i}(\xi) ; 2 \leq i \leq n\right\} \leq 0,
$$


we obtain

$$
\liminf _{\xi \rightarrow+\infty} \frac{\int_{0}^{T} \sup _{|x| \leq \xi} F(t, x) \mathrm{d} t}{\xi^{2}} \leq\left(\int_{0}^{T} b(t) \mathrm{d} t\right) \liminf _{\xi \rightarrow+\infty} \frac{F_{1}(\xi)}{\xi^{2}}<+\infty .
$$

Hence, the conclusion follows from Theorem 3.1.

Arguing as in the proof of Theorem 3.1, but using conclusion (c) of Theorem 2.4 instead of (b), one establishes the following result.

Theorem 3.4 Assume that Assumption (A1) holds. Furthermore, suppose that

(E1) $\liminf _{\xi \rightarrow 0^{+}} \frac{\int_{0}^{T} \sup _{|x| \leq \xi} F(t, x) \mathrm{d} t}{\xi^{2}}<\frac{C_{1}}{\left(A(\alpha)+\frac{2 T|a| \infty}{3}\right) k^{2} C_{2}} \lim \sup _{\xi \rightarrow 0^{+}} \frac{\int_{\frac{T}{4}}^{\frac{3 T}{4}} F(t, \xi) \mathrm{d} t}{\xi^{2}}$. Then, for each $\lambda \in] \lambda_{3}, \lambda_{4}[$ where

$$
\lambda_{3}:=\frac{\left(A(\alpha)+\frac{2 T\|a\|_{\infty}}{3}\right) C_{2}}{\lim \sup _{\xi \rightarrow 0^{+}} \frac{\int_{\frac{T}{4}}^{\frac{T}{4}} F(t, \xi) \mathrm{d} t}{\xi^{2}}}
$$

and

$$
\lambda_{4}:=\frac{C_{1}}{k_{2} \liminf _{\xi \rightarrow 0^{+}} \frac{\int_{0}^{T} \sup |x| \leq \xi}{\xi^{2}} F(t, x) \mathrm{d} t},
$$

for all continuous functions $I_{j}: \mathbb{R} \longrightarrow \mathbb{R}, j=1, \ldots, n$, for which $J_{j}(x)=\int_{0}^{x} I_{j}(\xi) \mathrm{d} \xi, j=1, \ldots, n$, for every $x \in \mathbb{R}$ are non-positive functions and satisfy the condition

$$
J_{0}:=\lim _{\xi \rightarrow 0^{+}} \frac{\int_{0}^{T} \sup _{|x| \leq \xi} G(t, x) \mathrm{d} t}{\xi^{2}}<+\infty,
$$

and for every $\mu \in\left[0, \mu_{J, \lambda}\left[\right.\right.$ where $\mu_{J, \lambda}:=\frac{C_{1}}{T k^{2} J_{0}}\left(1-\frac{\lambda k^{2}}{C_{1}} \liminf _{\xi \rightarrow 0^{+}} \frac{\int_{0}^{T} \sup _{|x| \leq \xi} F(t, x) \mathrm{d} t}{\xi^{2}}\right)$, the problem $\left(D_{\lambda, \mu}\right)$ has a sequence of pairwise distinct classical solutions which strongly converges to 0 in $E^{\alpha}$.

Proof Fix $\bar{\lambda} \in] \lambda_{3}, \lambda_{4}$ [ and let $I_{j}, j=1, \ldots n$, are the functions satisfying the condition (16). Since $\bar{\lambda}<\lambda_{2}$, one has $\mu_{J, \bar{\lambda}}>0$. Fix $\left.\bar{\mu} \in\right] 0, \mu_{J, \bar{\lambda}}\left[\right.$ and set $\nu_{3}:=\lambda_{3}$ and $\nu_{4}:=\frac{\lambda_{4} C_{1}}{C_{1}+\frac{\bar{\mu}}{\bar{\lambda}} \lambda_{4} T k^{2} J_{0}}$. If $J_{0}=0$, clearly, $v_{3}=\lambda_{3}, v_{4}=\lambda_{4}$, and $\left.\lambda \in\right] v_{3}, v_{4}\left[\right.$. If $J_{0} \neq 0$, since $\bar{\mu}<\mu_{J, \bar{\lambda}}$, one has

$$
\frac{\bar{\lambda}}{\lambda_{4}}+\frac{\bar{\mu} T k^{2} J_{0}}{C_{1}}<1
$$

and so

$$
\frac{C_{1} \lambda_{4}}{C_{1}+\frac{\bar{\mu}}{\bar{\lambda}} \lambda_{4} T k^{2} J_{0}}>\bar{\lambda}
$$

namely, $\bar{\lambda}<v_{4}$. Hence, recalling that $\bar{\lambda}>\lambda_{3}=v_{3}$, one has $\left.\bar{\lambda} \in\right] v_{3}, v_{4}[$. 
Now, put $Q(t, x)=F(t, x)-\frac{\bar{\mu}}{\bar{\lambda}} \sum_{j=1}^{n} J_{j}(x)$ for all $x \in \mathbb{R}$ and $t \in[0, T]$. Since

$$
\frac{\int_{0}^{T} \sup _{|x| \leq \xi} Q(t, x) \mathrm{d} t}{\xi^{2}} \leq \frac{\int_{0}^{T} \sup _{|x| \leq \xi} F(t, x) \mathrm{d} t}{\xi^{2}}+\frac{\bar{\mu}}{\bar{\lambda}} \frac{T \sup _{|x| \leq \xi} \sum_{j=1}^{n}-J_{j}(x)}{\xi^{2}}
$$

taking (16) into account, one has

$$
\liminf _{\xi \rightarrow 0^{+}} \frac{\int_{0}^{T} \sup _{|x| \leq \xi} Q(t, x) \mathrm{d} t}{\xi^{2}} \leq \liminf _{\xi \rightarrow 0^{+}} \frac{\int_{0}^{T} \sup _{|x| \leq \xi} F(t, x) \mathrm{d} t}{\xi^{2}}+\frac{\bar{\mu} T}{\bar{\lambda}} J_{0} .
$$

Moreover, since $J_{j}, j=1, \ldots, n$, are non-positive, we have

$$
\limsup _{\xi \rightarrow 0^{+}} \frac{\int_{\frac{T}{4}}^{\frac{3 T}{4}} Q(t, \xi) \mathrm{d} t}{\xi^{2}} \geq \limsup _{\xi \rightarrow 0^{+}} \frac{\int_{\frac{T}{4}}^{\frac{3 T}{4}} F(t, \xi) \mathrm{d} t}{\xi^{2}}
$$

Therefore, from (17) and (18), we obtain

$$
\bar{\lambda} \in] v_{3}, v_{4}[\subseteq] \frac{\left(A(\alpha)+\frac{2 T\|a\|_{\infty}}{3}\right) C_{2}}{\limsup _{|\xi| \rightarrow 0^{+}} \frac{\int_{\frac{T}{4}}^{\frac{3 T}{4}} F(t, \xi) \mathrm{d} t}{\xi^{2}}}, \frac{C_{1}}{k^{2} \liminf _{\xi \rightarrow 0^{+}} \frac{\int_{0}^{T} \sup _{|x| \leq \xi} F(t, x) \mathrm{d} t}{\xi^{2}}}[\subseteq] \lambda_{3}, \lambda_{4}[.
$$

We take $X, \Phi, \Psi$, and $I_{\bar{\lambda}}$ as in the proof of Theorem 3.1. We prove that $\delta<+\infty$. For this purpose, let $\left\{\xi_{n}\right\}$ be a sequence of positive numbers such that $\xi_{n} \rightarrow 0^{+}$as $n \rightarrow+\infty$ and

$$
\lim _{n \rightarrow \infty} \frac{\int_{0}^{T} \sup _{|x| \leq \xi_{n}} F(t, x) \mathrm{d} t}{\xi_{n}^{2}}<+\infty
$$

Put $r_{n}=\frac{C_{1} \xi_{n}^{2}}{k^{2}}$ for all $n \in \mathbb{N}$. Let us show that the functional $I_{\bar{\lambda}}$ has no local minimum at zero. For this purpose, let $\left\{\gamma_{n}\right\}$ be a sequence of positive numbers and $\tau>0$ such that $\gamma_{n} \rightarrow 0^{+}$ as $n \rightarrow \infty$ and

$$
\frac{1}{\bar{\lambda}}<\tau<\frac{\int_{\frac{T}{4}}^{\frac{3 T}{4}} F\left(t, \gamma_{n}\right) \mathrm{d} t}{\left(A(\alpha)+\frac{2 T\|a\|_{\infty}}{3}\right) C_{2} \gamma_{n}^{2}}
$$

for each $n \in \mathbb{N}$ large enough. Let $\left\{w_{n}\right\}$ be a sequence in $X$ defined by (12). So, owing to (13), (14), and (19) we obtain

$$
\begin{aligned}
I_{\bar{\lambda}}\left(w_{n}\right) & =\Phi\left(w_{n}\right)-\bar{\lambda} \Psi\left(w_{n}\right) \leq\left(A(\alpha)+\frac{2 T\|a\|_{\infty}}{3}\right) C_{2} \gamma_{n}^{2}-\bar{\lambda} \int_{\frac{T}{4}}^{\frac{3 T}{4}} F\left(t, \gamma_{n}\right) \mathrm{d} t \\
& <(1-\bar{\lambda} \tau)\left(A(\alpha)+\frac{2 T\|a\|_{\infty}}{3}\right) C_{2} \gamma_{n}^{2}<0
\end{aligned}
$$

for every $n \in \mathbb{N}$ large enough. Since $I_{\bar{\lambda}}(0)=0,0$ is not a local minimum of the functional $I_{\bar{\lambda}}$. Hence, the part (c) of Theorem 2.4 ensures that there exists a sequence $\left\{u_{n}\right\}$ in $X$ of critical points of $I_{\bar{\lambda}}$ such that $\left\|u_{n}\right\|_{a, \alpha} \rightarrow 0$ as $n \rightarrow \infty$, and the proof is complete. 
Remark 3.5 Applying Theorem 3.4, results similar to Remark 3.3, and Corollaries 3.2 and 3.3 can be obtained.

We end this paper by giving the following example as an application of Theorem 3.4.

Example 3.2 Let $\alpha=0.625, T=1, n=2, t_{1}=\frac{1}{3}, t_{2}=\frac{2}{3}, a(t)=\frac{1}{t+1}$ for all $t \in[0,1], f_{1}$ : $[0,1] \times(\mathbb{R} \backslash\{0\}) \longrightarrow \mathbb{R}$ be the function defined by

$$
\begin{aligned}
f_{1}(x)= & 2 x\left(\ln \left(\ln \left(\frac{1}{x^{2}}\right)\right)-\ln ^{-1}\left(\frac{1}{x^{2}}\right)\right) \sin ^{2}\left(\ln \left(\ln \left(\ln \left(\frac{1}{x^{2}}\right)\right)\right)\right) \\
& -4 x \ln ^{-1}\left(\frac{1}{x^{2}}\right) \sin \left(\ln \left(\ln \left(\ln \left(\frac{1}{x^{2}}\right)\right)\right)\right) \\
& +8 x \ln ^{-2}\left(\frac{1}{x^{2}}\right)\left(1+\ln \left(\frac{1}{x^{2}}\right)\right)
\end{aligned}
$$

and

$$
f(t, x)= \begin{cases}e^{t} f_{1}(x) & \text { if }(t, x) \in[0,1] \times(\mathbb{R} \backslash\{0\}) \\ 0 & \text { if }(t, x) \in[0,1] \times\{0\}\end{cases}
$$

and let $h(x)=\frac{\Gamma^{2}(0.625)}{32} \arctan x$, for all $x \in \mathbb{R}, I_{1}(x)=-\frac{x}{3}$, and $I_{2}(x)=-\frac{2 x}{3}$ for all $x \in \mathbb{R}$. A direct calculation shows

$$
F(t, x)= \begin{cases}e^{t} x^{2} \ln \left(\ln \left(\frac{1}{x^{2}}\right)\right) \sin ^{2}\left(\ln \left(\ln \left(\ln \left(\frac{1}{x^{2}}\right)\right)\right)\right)+4 x^{2} \ln ^{-1}\left(\frac{1}{x^{2}}\right), & \text { if }(t, x) \in[0,1] \times(\mathbb{R} \backslash\{0\}), \\ 0 & \text { if }(t, x) \in[0,1] \times\{0\} .\end{cases}
$$

According to the data above we have $k=2 \Gamma^{-1}(0.625), L=\frac{\Gamma^{2}(0.625)}{32}, C_{1}=0.25>0, C_{2}=0.75$, and

$$
\liminf _{\xi \rightarrow 0^{+}} \frac{\int_{0}^{1} \sup _{|x| \leq \xi} F(t, x) \mathrm{d} t}{\xi^{2}}=0 \text { and } \limsup _{\xi \rightarrow 0^{+}} \frac{\int_{\frac{1}{4}}^{\frac{3}{4}} F(t, \xi) \mathrm{d} t}{\xi^{2}}=+\infty .
$$

Hence, using Theorem 3.4, since

$$
J_{0}:=\lim _{\xi \rightarrow 0^{+}} \frac{\sup _{|x| \leq \xi}\left(-J_{1}(x)-J_{2}(x)\right)}{\xi^{2}}=\frac{1}{2}<\infty,
$$

the problem

$$
\begin{aligned}
& { }_{t} D_{1}^{0.625}\left({ }_{0}^{c} D_{t}^{0.625} u(t)\right)+\frac{u(t)}{t+1}=\lambda f(t, u(t))+\frac{\Gamma^{2}(0.526)}{32} \arctan (u(t)), \\
& t \neq \frac{1}{3}, t \neq \frac{2}{3} \text { a.e. } t \in[0,1], \\
& \Delta\left({ }_{t} D_{1}^{-0.375}\left({ }_{0}^{c} D_{t}^{0.625} u\right)\right)\left(\frac{1}{3}\right)=-\frac{\mu}{3}\left(u\left(\frac{1}{3}\right)\right), \\
& \Delta\left({ }_{t} D_{1}^{-0.375}\left({ }_{0}^{c} D_{t}^{0.625} u\right)\right)\left(\frac{2}{3}\right)=-\frac{2 \mu}{3}\left(u\left(\frac{2}{3}\right)\right) \\
& u(0)=u(1)=0,
\end{aligned}
$$


for every $(\lambda, \mu) \in] 0,+\infty\left[\times\left[0, \frac{\Gamma^{2}(0.625)}{32}[\right.\right.$ has a sequence of pairwise distinct classical solutions which strongly converges to 0 in $E^{0.625}$.

\section{Competing interests}

The authors declare that they have no competing interests.

Authors' contributions

All authors contributed to each part of this study equally and read and approved the final version of the manuscript.

\section{Author details}

${ }^{1}$ Department of Mathematics, Faculty of Sciences, Razi University, Kermanshah, 67149, Iran. ${ }^{2}$ Department of Economics, University of Messina, via dei Verdi, 75, Messina, Italy.

Received: 7 June 2016 Accepted: 11 July 2016 Published online: 25 July 2016

\section{References}

1. Diethelm, K, Freed, AD: On the solution of nonlinear fractional order differential equations used in the modeling of viscoplasticity. In: Keil, F, Mackens, W, Voss, H, Werther, J (eds.) Scientific Computing in Chemical Engineering II Computational Fluid Dynamics, Reaction Engineering and Molecular Properties, pp. 217-224. Springer, Heidelberg (1999)

2. Gaul, L, Klein, P, Kempfle, S: Damping description involving fractional operators. Mech. Syst. Signal Process. 5, 81-88 (1991)

3. Glockle, WG, Nonnenmacher, TF: A fractional calculus approach of self-similar protein dynamics. Biophys. J. 68, 46-53 (1995)

4. Hilferm, R: Applications of Fractional Calculus in Physics. World Scientific, Singapore (2000)

5. Kilbas, AA, Srivastava, HM, Trujillo, JJ: Theory and Applications of Fractional Differential Equations. Elsevier, Amsterdam (2006)

6. Mainardi, F: Fractional calculus: some basic problems in continuum and statistical mechanics. In: Carpinteri, A, Maniardi, F (eds.) Fractals and Fractional Calculus in Continuum Mechanics, pp. 291-348. Springer, Wien (1997)

7. Metzler, F, Schick, W, Kilan, HG, Nonnenmacher, TF: Relaxation in filled polymers: a fractional calculus approach. J. Chem. Phys. 103, 7180-7186 (1995)

8. Samko, SG, Kilbas, AA, Marichev, Ol: Fractional Integral and Derivatives: Theory and Applications. Gordon \& Breach, Longhorne (1993)

9. Lakshmikantham, V, Vatsala, AS: Basic theory of fractional differential equations. Nonlinear Anal. TMA 69, 2677-2682 (2008)

10. Miller, KS, Ross, B: An Introduction to the Fractional Calculus and Fractional Differential Equations. Wiley, New York (1993)

11. Podlubny, I: Fractional Differential Equations. Academic Press, San Diego (1999)

12. Zhou, Y: Basic Theory of Fractional Differential Equations, vol. 6. World Scientific, Singapore (2014)

13. Bai, C: Existence of three solutions for a nonlinear fractional boundary value problem via a critical points theorem. Abstr. Appl. Anal. 2012, Article ID 963105 (2012)

14. Bai, C: Existence of solutions for a nonlinear fractional boundary value problem via a local minimum theorem. Electron. J. Differ. Equ. 2012, 176 (2012)

15. Bai, C: Infinitely many solutions for a perturbed nonlinear fractional boundary value-problem. Electron. J. Differ. Equ. 2013, $136(2013)$

16. Chen, J, Tang, XH: Existence and multiplicity of solutions for some fractional boundary value problem via critical point theory. Abstr. Appl. Anal. 2012, Article ID 648635 (2012)

17. Ferrara, M, Molica Bisci, G: Remarks for one-dimensional fractional equations. Opusc. Math. 34(4), 691-698 (2014)

18. Galewski, M, Molica Bisci, G: Existence results for one-dimensional fractional equations. Math. Methods Appl. Sci. 39 1480-1492 (2016)

19. Graef, JR, Kong, L, Kong, Q: Multiple solutions of systems of fractional boundary value problems. Appl. Anal. 94 1288-1304 (2015)

20. Graef, JR, Kong, L, Kong, Q, Wang, M: Fractional boundary value problems with integral boundary conditions. Appl. Anal. 92, 2008-2020 (2013)

21. Heidarkhani, S: Multiple solutions for a nonlinear perturbed fractional boundary value problem. Dyn. Syst. Appl. 23 317-332 (2014)

22. Heidarkhani, S: Infinitely many solutions for nonlinear perturbed fractional boundary value problems. In: Annals of the University of Craiova. Math. Comp. Sci. Series, vol. 41, pp. 88-103 (2014)

23. Jiao, F, Zhou, Y: Existence of solutions for a class of fractional boundary value problems via critical point theory. Comput. Math. Appl. 62, 1181-1199 (2011)

24. Kong, L: Existence of solutions to boundary value problems arising from the fractional advection dispersion equation Electron. J. Differ. Equ. 2013, 106 (2013)

25. Molica Bisci, G: Fractional equations with bounded primitive. Appl. Math. Lett. 27, 53-58 (2014)

26. Zhou, Y, Peng, L: On the time-fractional Navier-Stokes equations. Comput. Math. Appl. (2016). doi:10.1016/j.camwa.2016.03.026

27. Lakshmikantham, V, Bainov, DD, Simeonov, PS: Theory of Impulsive Differential Equations. Series in Modern Applied Mathematics, vol. 6. World Scientific, Teaneck (1989)

28. Bainov, D, Simeonov, P: Systems with Impulse Effect. Ellis Horwood Series: Mathematics and Its Applications. Ellis Horwood, Chichester (1989) 
29. Benchohra, M, Henderson, J, Ntouyas, S: Theory of Impulsive Differential Equations. Contemporary Mathematics and Its Applications, vol. 2. Hindawi Publishing Corporation, New York (2006)

30. Heidarkhani, S, Ferrara, M, Salari, A: Infinitely many periodic solutions for a class of perturbed second-order differential equations with impulses. Acta Appl. Math. 139, 81-94 (2015)

31. Liu, X, Willms, AR: Impulsive controllability of linear dynamical systems with applications to maneuvers of spacecraft. Math. Probl. Eng. 2, 277-299 (1996)

32. Samoilenko, AM, Perestyuk, NA: Impulsive Differential Equations. World Scientific, Singapore (1995)

33. Bonanno, G, Rodríguez-López, R, Tersian, S: Existence of solutions to boundary-value problem for impulsive fractional differential equations. Fract. Calc. Appl. Anal. 3, 717-744 (2014)

34. De la Sen, M: On Riemann-Liouville and Caputo impulsive fractional calculus. In: Proc. of the World Congress on Engineering 2011, Vol. 1, London, UK (2011)

35. Tian, Y, Bai, Z: Existence results for the three-point impulsive boundary value problem involving fractional differential equations. Comput. Math. Appl. 59, 2601-2609 (2010)

36. Wang, J, Feckan, M, Zhou, Y: On the new concept of solutions and existence results for impulsive fractional evolution equations. Dyn. Partial Differ. Equ. 58, 345-361 (2011)

37. Ait Dads, E, Benchohra, M, Hamani, S: Impulsive fractional differential inclusions involving fractional derivative. Fract. Calc. Appl. Anal. 12(1), 15-38 (2009)

38. Bai, C: Impulsive periodic boundary value problems for fractional differential equation involving Riemann-Liouville sequential fractional derivative. J. Math. Anal. Appl. 384, 211-231 (2011)

39. Bai, C: Solvability of multi-point boundary value problem of nonlinear impulsive fractional differential equation at resonance. Electron. J. Qual. Theory Differ. Equ. 2011, 89 (2011)

40. Ke, TD, Lan, D: Decay integral solutions for a class of impulsive fractional differential equations in Banach spaces. Fract. Calc. Appl. Anal. 17(1), 96-121 (2014)

41. Rodríguez-López, R, Tersian, S: Multiple solutions to boundary value problem for impulsive fractional differential equations. Fract. Calc. Appl. Anal. 17, 1016-1038 (2014)

42. Heidarkhani, S, Salari, A: Nontrivial solutions for impulsive fractional differential systems through variational methods. Comput. Math. Appl. (2016). doi:10.1016/j.camwa.2016.04.016

43. Heidarkhani, S, Zhao, Y, Caristi, G, Afrouzi, GA, Moradi, S: Infinitely many solutions for perturbed impulsive fractional differential systems. Appl. Anal. (2016). doi:10.1080/00036811.2016.1192147

44. Heidarkhani, S, Zhou, Y, Caristi, G, Afrouzi, GA, Moradi, S: Existence results for fractional differential systems through a local minimization principle. Comput. Math. Appl. (2016). doi:10.1016/j.camwa.2016.04.012

45. Zhao, Y, Chen, H, Qin, B: Multiple solutions for a coupled system of nonlinear fractional differential equations via variational methods. Appl. Math. Comput. 257, 417-427 (2015)

46. Zhao, Y, Chen, H, Zhang, Q: Infinitely many solutions for fractional differential system via variational method. J. Appl. Math. Comput. 50, 589-609 (2016)

47. Bonanno, G, Molica Bisci, G: Infinitely many solutions for a boundary value problem with discontinuous nonlinearities. Bound. Value Probl. 2009, Article ID 670675 (2009)

48. Ricceri, B: A general variational principle and some of its applications. J. Comput. Appl. Math. 113, 401-410 (2000)

49. Bonanno, G, D'Aguì, G: On the Neumann problem for elliptic equations involving the $p$-Laplacian. J. Math. Anal. Appl. 358(358), 223-228 (2009)

50. Bonanno, G, Di Bella, B: Infinitely many solutions for a fourth-order elastic beam equation. NoDEA Nonlinear Differ. Equ. Appl. 18, 357-368 (2011)

51. Bonanno, G, Molica Bisci, G: A remark on perturbed elliptic Neumann problems. Stud. Univ. Babeş-Bolyai, Math. LV, 4 (2010)

52. Molica Bisci, G, Rădulescu, V: Ground state solutions of scalar field fractional Schrödinger equations. Calc. Var. 54, 2985-3008 (2015)

53. Molica Bisci, G, Rădulescu, V: Multiplicity results for elliptic fractional equations with subcritical term. Nonlinear Differ. Equ. Appl. 22, 721-739 (2015)

54. Molica Bisci, G, Servadei, R: A bifurcation result for non-local fractional equations. Anal. Appl. 13, 371-394 (2015)

55. Pucci, P, Serrin, J: Extensions of the mountain pass theorem. J. Funct. Anal. 59, 185-210 (1984)

56. Pucci, P, Serrin, J: A mountain pass theorem. J. Differ. Equ. 60, 142-149 (1985)

\section{Submit your manuscript to a SpringerOpen ${ }^{\circ}$ journal and benefit from:}

- Convenient online submission

Rigorous peer review

- Immediate publication on acceptance

- Open access: articles freely available online

- High visibility within the field

- Retaining the copyright to your article 